\title{
Towards international consensus on patient harm: perspectives on pressure injury policy
}

\begin{abstract}
Aims - To analyse influential policies that inform practice related to pressure injury management in Australia, England, Hong Kong, New Zealand, Scotland, and the United States of America.
\end{abstract}

Background - Pressure injuries are associated with significant harm to patients, and carry economic consequences for the health sector. Internationally, preventing and managing pressure injuries is a key nursing activity and quality indicator.

Evaluation - Comparative review and synthesis of pressure injury policies that inform practice.

Key issues - The predominant focus of policy is on patient risk assessment, compliance with documentation and pressure relief. Financial penalty for institutions is emerging as a strategy where pressure injuries occur. Comparisons of prevalence rates are hampered by the lack of consensus on data collection and reporting. To date there has been little evaluation of policy implementation and implemented policy strategies, associated guidelines remain founded upon expert opinion and low-level evidence.

Implications for nursing management - The pressure injury policy agenda has fostered a discourse of attention to incidents, compliance and penalty (sanctions). Prevention and intervention strategies are informed by technical and biomedical interpretations of patient risk and harm, with little attention given to the nature or design of nursing work. Considerable challenges remain if this policy agenda is to successfully eliminate pressure injury as a source of patient harm.

\section{Keywords}

nursing, pressure injury, pressure ulcer, patient safety, patient harm, policy analysis, regulation

\section{Introduction}

The aim of this study was to identify the core elements of international policies pertaining to pressure injury (PI), and synthesize progress toward the elimination of patient harm from PI . For the purpose of the review, policy is defined as a plan that steers action and investment (Cheung et al. 2010a) to reduce or prevent PI. Such policies are evidenced through directing 
statements of intent and clinical practice guidelines that are adopted to guide clinical decision making and link evidence with practice (Walt et al. 2008).

Several methodologies have been developed for the review of policy. In general, policy analysis seeks to establish the goals or problem that the policy seeks to address; the causal assumptions and expected benefits of the policy; and, opportunities to implement the policy (Cheung et al. 2010a). A more contextual approach to policy analysis, and the one adopted for the current analysis, presumes that policies are framings that give shape to particular foci and responses to a problem (Coveney 2010). By asking questions about how the problem is represented within policy, the assumptions and presuppositions considered problematic can be brought out more clearly (Bacchi 2012).

The need to conduct the analysis stems from the fact that, despite continued concern and attention to reducing the prevalence of pressure damage, reports suggest that interventions to ameliorate the problem may not achieve sustained results. The rate of preventable hospitalacquired PI fluctuates, and in some instances has increased (Mulligan et al. 2011). Given the prevalence and costs associated with PI, it is timely to undertake an international policy analysis to identify how PI regulation is positioned in various countries, including the nature of prevalence reporting, the types of strategies and interventions aimed at reducing the incidence of PI, and the regulatory strategies aimed at ensuring compliance with PI policies and the implications for nursing practice and leadership.

\section{Background}

Preventing and managing PI is a key nursing activity across all care settings and is recognised across international jurisdictions as an indicator of care quality (Montalvo 2007). Pressure injury remains a significant source of physical and emotional harm to patients. Individuals who experience hospital-acquired PI have been shown to have higher mortality, both within hospital and within 30 days of discharge (Lyder et al. 2012). In the United Kingdom, PI is identified as the highest burden of harm to patients (HSCIC 2014). These injuries are considered to be a (largely) preventable form of patient harm. Interventions related to PIs are associated with patient burden and have an impact on health-related quality of life (Gorecki et al. 2009). Internationally, PIs are associated with major personal costs to patients and the health sector (Dealey et al. 2012). In 2012 it was established that the cost of treating a PI in the United Kingdom (UK) ranged from £1214-14108 per annum; higher costs are reflected 
by increased healing time and greater risk of complications with more severe injuries (Dealey, Posnett \& Walker, 2012). In 2009 the estimated annual costs to the National Health Service (NHS) were $£ 2.64$ billion (Riordan \& Voegeli 2009), or in the order of $2 \%$ of the entire NHS budget (Bennett et al. 2004). Internationally, costs are comparable. For example in Australia, the most recently available figures collated by Nguyen, Chaboyer and Whitty (2015) estimate the cost of PI in 2012-3 to be $\$ 983$ million per annum, with 524,661 bed days lost.

Despite the prevalence and costs of PI, there is a paucity of robust or large-scale evidence to inform prevention (Nguyen et al. 2015). The evidence for improvement in hospital acquired PI rates is largely limited to quality improvement projects, with few randomized controlled trials. Indeed, much of the nursing literature to date reports knowledge and attitudes of nurses towards PI prevention (see for example: (Nguyen et al. 2015, Simonetti et al. 2015) rather than interventional studies aiming to reduce the prevalence and suffering associated with these injuries. Evidence-based clarity is still lacking in relation to best practice in both PI management and prevention. For example, patient repositioning is a staple nursing strategy for both prevention and treatment of PI. However, recent Cochrane reviews reveal a lack of evidence for repositioning as a prevention (Gillespie et al. 2014, Moore \& Cowman 2015). Additionally, while there is compelling evidence that PI occurs outside of hospital and older person care settings (Jones 2013), most prevalence studies and evaluations are hospital-based. There is a need for more robust research about PI prevention and management across a range of clinical contexts.

Predicting those at risk of developing a PI remains a nursing challenge, with assessment tools providing imperfect predictors of risk (García-Fernández et al. 2014b). A number of risk assessment tools are widely recommended as an arm of preventive strategies. PI risk assessment scales have been developed for differing patient populations, and to date, none have been found to be effective in all patient populations, or in all health care settings (Kelechi et al. 2013). Furthermore, issues have been raised about the validity and adequacy of these tools. For example, the Braden Scale has been found to be a poor predictor of PI risk in surgical patients or during acute illness (He et al. 2012). The low sensitivity and specificity of this tool was highlighted by Mulligan (2011) who reported that $50 \%$ of hospital patients with a PI were assessed as low risk utilising the Braden Scale. Despite this, the use of this scale by nurses has been advocated, believing it would help enable a focus on PI prevention strategies. 
The Waterlow scale is reported as having low interrater reliability (Kelechi et al. 2013). Despite these issues with individual tools, it has been argued that the use of a PI risk assessment scale heightens awareness of risk factors and patients at risk, and ensures assessment aimed at prevention is occurring (Kelechi et al. 2013).

\section{The PI policy agenda}

Internationally, many countries have developed policies and guidelines aimed at reducing the prevalence of PI. There are various approaches taken to these policies and guidelines, and some jurisdictions have introduced financial penalties or rewards associated with PI incidence (Sen et al. 2009). In order for comparative analysis of the prevalence and burden of harm, and to establish the effectiveness of interventions, a consistent definition of PI is required. A range of terms have been used to describe this form of tissue injury. Common terms have included bed or pressure sore, decubitus ulcer, pressure necrosis and ischemic ulcer. More recently there has been a move towards using the term pressure injury (AWMA 2012, National Pressure Ulcer Advisory Panel (NPUAP) 2014). Consensus exists on staging injury to the skin and underlying tissue, with this staging system employed widely in reporting systems (NPUAP 2014). Internationally, data collection on the incidence of PI remains limited or patchy. Whilst some jurisdictions (such as Australia) report incidence and prevalence at facility, state and national levels, in 2013 only five countries (Canada, Finland, Iceland, Norway and Portugal) reported data on PI to the Organisation for Economic Cooperation and Development (OECD).

\section{PI and patient experiences}

Adhering to targets for PI reduction involves meticulous documentation of whether PIs are acquired in a healthcare institution or the patient is admitted with a PI - the latter often referred to as being an 'inherited' PI. Severity or grading of PI is also commonly recorded. However, despite the surveillance of prevalence and severity of PI, the experiences and perspectives of patients themselves tend to be rather less scrutinised. Pain emerged as a significant issue for a small sample of hospital patients who felt nurses did not adequately recognise or treat the pain and discomfort associated with PI, and that devices aimed at relieving pressure sometimes caused patients additional discomfort and distress (Spilsbury et al. 2007). Gorecki et al. (2012) found similar concerns regarding pain and pressure relieving devices in a qualitative study of 30 patients from England and Northern Ireland. Additionally, these participants reported concern regarding inconsistent management of PI by health 
professionals and a lack of continuity of care. Some participants felt that their knowledge of their own PI was discounted by health professionals which discouraged future collaboration (Gorecki et al. 2012). Aside from prevention, there is little known about strategies that can reduce suffering associated with PI and PI interventions.

\section{Evaluation}

To provide a richer understanding of PI policy, and to uncover different or contested views on what is central or important in PI policy, we analysed policy documents informing care across the full range of health services from six countries. A convenience sample of policy documents was identified as the comparative analyssi was not intended to be world-wide and it was not feasible to locate an exhaustive collection of documents. Policy documents related to PI were located using relevant health service and professional association websites in the six countries represented by the team of collaborators. In addition, searches were performed of the Cochrane, National Institute for Health and Care Excellence (NICE) and Joanna Briggs Institute databases. Field experts were also contacted to identify documents not identified through this search. Documents were selected because they were primary national-level policy documents related to PI. Employing a modified set of criteria derived by Cheung, Mirzaei and Leeder (2010b) we examined the policy background; policy goals; resources; monitoring and evaluation; and obligations (including penalties). To allow for comparative analysis (Weimer and Vining 2015), relevant narrative data was extracted from each document, and coded against these criteria. This process of coding and extracting relevant components of narrative description allowed us to explore how the various components of PI policy were represented across the sample of policy documents. This process allowed for identification consistency, similarities and identification of gaps and inconsistencies (Ritter et al., 2016). To ensure consistency across the process two authors cross-checked the mapping and analysis, with differences resolved through discussion and consensus. From this analysis a narrative synthesis of PI policy documents was derived.

**Insert table 2 here**

\section{Results}


The search yielded 7 national-level policy or standards documents and associated practice guidelines. Two of the policy were multi-country collaborations (Table 1). The primary objectives of the policies reviewed were to summarise evidence and provide guidance to clinicians on the prevention and management of PI. The goals of the policies almost exclusively focus upon PI risk assessment, nutritional assessment and intervention for those at risk, relief or redistribution of pressure, and optimal wound management. A number are predominately focused upon treatment approaches, with less attention given to prevention.

\section{Policy Background}

The publication date for the policies included in the review ranged from 2009 to 2014, with a number in their second (WOCN. 2010, NPUAP 2014) or third iterations (QIS 2009). The main driver for policy development was the need to provide guidance for clinicians regarding the prevention and management of PI. In the UK, PI prevalence statistics and economic modelling were employed to underpin the need for concerted policy action to address the cost burden of PI on health services (NICE 2014). Reflecting concern for harm to patients, policy has increasingly included a focus upon quality, safety and reducing harm.

The work of developing PI policy has been largely undertaken by expert panels drawn from not-for-profit professional organisations and Government bodies. Outside of the UK, a significant component of policy development was funded by wound management associations (New Zealand, Australia, Hong Kong, United States) who sought to influence public policy at a national level. A consistent trend over the period of the policy review was the development of policy through collaborative efforts between national interest groups and expert panels. Expert panels were largely constituted by nurse experts in tissue viability and wound management, with minimal evidence of consumer involvement.

Collaboration across a number of the jurisdictions initially produced multi-country policies such the American National Pressure Ulcer Advisory Panel (NPUAP) and the European Pressure Ulcer Advisory Panel (EPUAP). The move towards these multi-country collaborations has supported the review of extensive evidence, large scale collaboration and increased the rigour of the review process and the scope of recommendations. An outcome of these collaborations has been agreement on classification systems with a view to standardising international reporting of PI incidence and prevalence. 


\section{Evidence and PI policy}

As policies have evolved, attention has been drawn to the need for a research agenda that provides an evidence base for prevention and management of PI. However, few goals in the policies reviewed are explicit or measurable. Throughout the body of policy, the majority of recommendations are founded upon expert opinion rather than empirical evidence, with only a small proportion founded upon mid-range or high level evidence. Illustrative of the absence of sound evidence, the Pan Pacific Clinical Practice Guidelines (2014) report that the majority of the recommendations made (42\%) are based on expert consensus in the absence of sufficient evidence, with $9 \%$ assessed to have good levels of supportive evidence, and $11 \%$ strong levels of evidence. The grounding of guidelines in contemporary evidence was also limited in some of the policies reviewed, with one relying on evidence from systematic reviews that had been published ten years before the release of the policy (NHS QIS, 2009).

\section{Policy Implementation, Monitoring and Evaluation}

In the majority of country-level policies examined in the review, each jurisdiction has implemented an additional policy and/or guideline that operationalised the national overarching policy and adapted it to particular regional or facility contexts. Another approach has been to operationalise the overarching policy via the provision of implementation tools and resources; rather than through an additional policy. As a result, a wide array of online resources are available to clinicians.

Strategies for monitoring and evaluation of policy implementation are not specifically contained within the policy documents, and no evaluation mechanisms are addressed or mandated. Action to demonstrate policy implementation can be inferred through recommendations regarding the documentation of action in patient notes (such as Scotland and England). Similarly no specific outcome measures are identified in the policy documents. At a country level, parallel to PI policy and guidelines, a number of jurisdictions (Australia, Hong Kong, United States, England and Scotland) have developed quality assurance and audit mechanisms that capture the intent of PI policies. In these countries, specific standards have been developed to address the quality and safety aspects of PI, and facility accreditation is contingent upon meeting these standards. Furthermore, in the United States the National Quality Forum (NQF) has identified hospital acquired stage III and IV PI as "never 
events"(AHRQ). In this context, stage III and IV PIs are categorised along with 28 other events by the NQF as events that should never occur during hospital admission.

Although international consensus has emerged in recent years in the PI policy area on definitions and grading of PI, there is less consistency across countries on data collection and the reporting PI incidence and prevalence. Reporting varies from not formally required (New Zealand and Scotland) to institutions being required to have reporting systems in place to achieve accreditation (Australia). A less common feature of PI policy context is the mandated reporting of outcome and assessment data (United States). Table 2 provides a comparative summary of the PI policies reviewed.

** Insert table 2 here**

\section{Obligations and Penalties?}

Reflecting the development of policy largely by professional organisations, obligations and penalties are not a feature of PI policy. Similar to the quality assurance mechanisms that have been developed in parallel to PI policy development, there is an emerging trend towards penalty for hospital acquired PI. In Australia, one state has recently introduced a system of financial penalty for development of severe PI in hospitalised patients (Walker, Huxley, Juttner et al 2014). The situation is similar for private providers in Australia, with the private insurer Medibank listing stage III and IV PIs as an adverse event for which it seeks not to pay benefits to private hospitals (Medibank 2015). Similarly in the United States, the Centres for Medicare and Medicaid Services (CMS) do not fund hospital-acquired PI (Sen et al. 2009).

\section{Resources}

Reflecting the primary focus on providing guidance for clinicians in the management and prevention of PU and the development of policy by professional organisations, the body of policy gives little attention to investment to support action. The primary human resource issue addressed in the policy is the need for training in assessment, preventive measures and wound management. Organizational capacity, infrastructure, workforce and service redesign are not factors addressed to underpin the goals of the PI policies.

\section{Discussion}


In the jurisdictions reviewed, the initiators of PI policy were primarily professional associations, with clinical nurse experts driving forward this agenda. The fundamental premise of much of this work has been the development of policy and clinical practice guidelines to promote consistency in practice and inform decisions and clinical judgment through evidence.

It is notable that the focus of PI policy remains largely unchanged since the Agency for Health Care Policy and Research released its policy more than fifteen years ago. The predominant focus of policy over this period has been interventions to reduce pressure or manage PI when they develop. Following the work of Braden and Bergstrom (1987) the interpretation of pressure or decreased tissue tolerance as the primary etiological factor for PI has promulgated an "end-point" interpretation of the factors that contribute to PI development.

This end point framework of causation overlooks the broader care and system-level factors that may contribute to risk of PI and result in pressure and decreased tissue tolerance. The body of policy analysed for this review highlights that attention remains focused upon the technical aspects of relieving pressure or promoting wound healing and technical or biomedical interpretations of patient risk and harm. This focus on pressure is likely to have arisen from wound management interpretations of PI, largely driven forward by wound management associations. Garcia-Fernandez et al., (2014a) have theorised that PI occur in individuals with some type of dependence, who are unable to care for themselves and are dependent on others for care. Reframing the underlying causative factor as "dependence" rather than "pressure" draws into focus areas of nursing practice not canvassed in any detail in the current body of policy. Moreover, the capacity of patients for active involvement in their own risk prevention remains overlooked.

\section{The nursing work environment and design of nursing work}

Considerable research highlights the link between the nursing work environment and nursing workforce and patient safety and quality (Zhu et al. 2012), with evidence that these factors influence PI incidents and prevalence (Stone et al. 2007, Horn et al. 2005). Given the substantial body of evidence that confirms the profile of the nursing workforce influences nurse sensitive patient outcomes, it is notable that the body of PI policy reviewed has given little attention to staffing or other human resource contextual factors. The focus of policy 
remains at the individual patient-level. As a consequence, recommended interventions have given priority to screening patients for risk and implementing biomechanical interventions to reduce or redistribute pressure.

Highlighting how the nursing work environment influences PI risk, in one Australian study (Mulligan2011) reported that a contributing factor in PI prevalence was bed management strategies and the impact of these practices on patient care. Mulligan (2011) reported that these administrative strategies increased the number of "location moves" patients experienced during hospitalisation. These moves intensified nursing work, fragmented care, and adversely affected continuity of care by shifting the emphasis from patient-centred care to bed management and patient flow (Mulligan (2011). Similarly, analysis of PI prevalence data for the period 2005-2011 in Germany indicated that the number of full-time employees in nursing homes had an influence on the incidence of PI (Heinhold et al. 2014). Examining the influence of nurse staffing on PI and associated interventions Sving et al (2014) reported that, when total hours of nursing care was lower, patients were more likely to have pressurereducing mattresses implemented, but were less likely to have planned repositioning. Whereas, employing a retrospective process-tracing case study method Pinkey et al (Pinkney et al. 2014) examined eight cases where individuals had developed a category 3 or 4 PI. Through detailed reconstruction of the cases the organisational context was revealed as a significant contributing factor in the development of these PI. Specifically, clinicians failed to listen to the patients' or carers'; clinicians did not recognise or respond to clear signs of an existing PI or to the risk of developing one; and services were not effectively coordinated. Reflection on the findings of these studies suggest that skill mix, nurse staffing and perceived staffing adequacy may potentially be sensitive predictors of PI occurrence.

\section{The challenge of providing evidence to inform policy and practice}

Despite the prevalence, costs and harm directly associated with PI, there is a paucity of largescale or robust evidence to inform preventive policy and guide action. The majority of the recommendations in the clinical practice guidelines reviewed are founded on expert opinion or low-level evidence. In addition, there were regional inconsistencies in policy across countries and between countries (i.e. England and Scotland). In the absence of country-level funding and research foci, much of the published research employed in developing policy and 
guidelines is underpowered, and many assumptions or common practices have little empirical substantiation.

There remains a lack of consensus around data collection and reporting, which contributes to considerable variability in data reporting across jurisdictions. This results in limited capacity for comparison across countries and contributes to potential reporting bias. In Australia, data collection on PI remains underdeveloped and available data presents a patchy picture on the burden from PI for patients, the health system, and broader society. In the U.S. no established standard has been implemented to guide consistent identification of PI (Zaratkiewicz et al. 2010). These gaps limit data available regarding incidence, assessment and management and support a need for universal tracking mechanisms. Furthermore, the prevalence of PI in community or informal care and social service settings has been given little attention (Nguyen et al. 2015). The burden of PI occurring outside of hospital and residential care settings no doubt represents a substantial weight of patient harm as well as economic costs.

Inconsistency between hospital coding systems and PI classification systems has also limited the capacity to identify the mechanisms for PI development (Pan Pacific 2012). Similarly variations in reporting have limited international comparisons on prevalence. Another area of controversy is the concept of PI avoidability. Expert consensus suggests that not all PI are avoidable and acknowledges there are patient situations where pressure cannot be relieved and perfusion cannot be improved (Black et al. 2011). The definition of avoidable PI are those that develop in the absence of assessment or intervention (NHS \& NPUAP). The contestability of this definition is highlighted in case reviews which have for example reported that, during a one year period in five acute NHS trusts (UK), only $43 \%$ of PIs sustained were deemed avoidable (Downie et al. 2013).

\section{Implications for nursing management}

Considerable challenges remain if this policy agenda is to successfully eliminate pressure injury as a source of patient harm. Moving from a focus of attention to reporting and monitoring incidents, nurse managers should now turn attention to fostering and supporting innovation in the delivery and design of nursing work. As a priority nurse managers and leaders ought to give considered attention to the implications of nursing skill mix and the design of nursing work and work flow upon PI causation in policy and guidelines. It has now 
been more than a decade since the association was first confirmed between nurse staffing and nursing education levels and patient mortality or poorer clinical outcomes (Lang et al. 2004, Aiken et al. 2003). Yet, sparse consideration has been given in PI policy to the influence of the broader nursing work environment or organisational context and whether this contributes to risk of PI, or contributes to factors that increase the risk of PI. Similarly, little attention has been given to the nature of nursing work, or whether the design of nursing work, and the conceptualisation of harm and risk perpetuates risk of patient harm from pressure. To advocate for safer patient and nursing work environments, forms of nursing leadership focused upon developing just work cultures are necessary to influence safety outcomes (Squires et al., 2010)

It is of significance to note that U.S. policy on PI as a "never event" has spurred reexamination of clinical practices and a shift to address system approaches to implement evidence-based strategies to address avoidable PI. Rau (2014) reported that $14 \%$ of hospitals in the U.S. anticipated that their Medicare funding would be reduced by $1 \%$ on the basis of high rates of hospital acquired conditions (including PI).CMS expects to provide hospitals with information about the calculation of their HAC score for the fiscal year 2016 adjustment in late summer 2015. The Centers for Medicare and Medicaid Services (CMS) expect that up to $25 \%$ of hospitals will be subject to the penalty, and over time there are plans to increase the number events measured (Health Policy Brief 2015). In recent years evidence has emerged on the benefits of nurse rounding and other forms of work re-design (Dyck et al. 2013); however, this evidence remains absent from PI policy and guidelines. If patients' journeys through the health care system are to be safe and PI free, it is important to address the various components of the system and their inter-linkages.

It is clear that multicomponent initiatives (e.g., "bundling" interventions) are needed to address PI prevention as a system-wide safety initiative (Sullivan and Schoelles, 2013). Prior systematic reviews identify the need to examine daily care processes as another care facet that has had limited focus in reducing avoidable PI's (Sullivan \& Schoelles 2013, Niederhauser et al. 2012, Soban et al. 2011). Recent studies using a "turn team" (Still et al. 2013) or prophylactic use of wound dressings to prevent PI caused by medical devices (Black et al. 2013) exemplify the evolving focus on daily care processes that need to be integrated into daily care practices to prevent PI's. Policy tied with penalties to for "never event" occurrence is a driving force to innovate practice. 


\section{Conclusions:}

The PI policy agenda has fostered a discourse of attention to patient incidents, compliance and more recently, financial penalty. Yet the occurrence of PI outside of the hospital setting, patient capacity for involvement, the work environment, nursing workforce and skill mix, and system contributions to the development of inconsistency in assessment and treatment of PI has been largely excluded from consideration. The analysis highlights the significant challenges that remain for nurses, particularly nurse leaders, if this policy agenda is to eliminate patient harm from PI. There is an urgent need for nurse researchers to focus upon robust interventional studies that seek to identify nurse-led or nurse-focused strategies to reduce the prevalence and suffering associated with PI. Moving beyond attention to the biomechanics of PI aetiology, there is a need to adopt whole of systems approaches to understanding the factors in the nursing work environment that sustain risk and appropriate mitigation strategies.

\section{References:}

AHRQ (2015) Patient safety primer: never events. Accessed $29^{\text {th }}$ September 2015: http://psnet.ahrq.gov/primer.aspx?primerID $=3$

Aiken L.H., Clarke S.P., Cheung R.B., Sloane D.M. \& Silber J.H. (2003) Educational Levels of Hospital Nurses and Surgical Patient Mortality. JAMA : the journal of the American Medical Association, 290(12), 1617-1623.

AWMA (2012) Australian Wound Management Association. Pan Pacific clinical practice guideline for the prevention and management of pressure injury. (A.W.M. Association ed.). Cambridge Publishing, Osborne Park, WA.

Bacchi C. (2012) Introducing the 'What's the Problem Represented to be?' approach In Engaging with Carol Bacchi: strategic interventions and exchanges (A. Bletsas \& C. Beasley eds.). The University of Adelaide Press The Univeristy of Adelaide, South Australia.

Bennett G., Dealey C. \& Posnett J. (2004) The cost of pressure ulcers in the UK. Age and ageing, 33(3), 230-235.

Bergstrom N., Braden B.J., Laguzza A. \& Holman V. (1987) The Braden Scale for Predicting Pressure Sore Risk. Nurs Res, 36(4), 205-210.

Black J., Alves P., Brindle C.T., Dealey C., Santamaria N., Call E. \& Clark M. (2013) Use of wound dressings to enhance prevention of pressure ulcers caused by medical devices. Int Wound J. 12(3):322-7. doi: 10.1111/iwj.12111Jul 1.

Black J., Edsberg L., Baharestani M., Langemo D., Goldverg M., McNichol L. \& Cuddigan J. (2011) Pressure ulcers: avoidable or unavoidable? Results of the National Pressure Ulcer Advisory Panel consensus conference. Ostomy Wound Management, 57(2), 2437.

Cheung K.K., Mirzaei M. \& Leeder S. (2010a) Health policy analysis: a tool to evaluate in policy documents the alignment between policy statements and intended outcomes. Australian Health Review, 34, 405-413. 
Cheung K.K., Mirzaei M. \& Leeder S. (2010b) Health policy analysis: a tool to evaluate in policy documents the alignment between policy statements and intended outcomes. Australian Health Review, 34(4), 405-413.

Coveney J. (2010) Analyzing public health policy: three approaches. Health promotion practice, 11(4), 515-521.

Dealey C., Posnett J. \& Walker A. (2012) The cost of pressure ulcers in the United Kingdom. $J$ Wound Care, 21(6), 261-266.

Downie F., Guy H., Gilroy P., Royall D. \& Daview S. (2013) Are 95\% of hospital-acquired pressure ulcers avoidable? Wounds $U K$, 9(3), 16-22.

Dyck D., Thiele T., Kebicz R., Klassen M. \& Erenberg C. (2013) Hourly rounding for falls prevention: a change initiative. Creative nursing, 19(3), 153-158.

García-Fernández F.P., Agreda J.J.S., Verdú J. \& Pancorbo-Hidalgo P.L. (2014a) A New Theoretical Model for the Development of Pressure Ulcers and Other DependenceRelated Lesions. Journal of Nursing Scholarship, 46(1), 28-38.

García-Fernández F.P., Pancorbo-Hidalgo P.L. \& Agreda J.J.S. (2014b) Predictive capacity of risk assessment scales and clinical judgment for pressure ulcers: a meta-analysis. Journal of Wound Ostomy \& Continence Nursing, 41(1), 24-34.

Gillespie B.M., Chaboyer W.P., McInnes E., Kent B., Whitty J.A. \& Thalib L. (2014) Repositioning for pressure ulcer prevention in adults. Cochrane Database Syst Rev, 7(4).

Gorecki C., Brown J.M., Nelson E.A., Briggs M., Schoonhoven L., Dealey C., Defloor T. \& Nixon J. (2009) Impact of pressure ulcers on quality of life in older patients: a systematic review. J Am Geriatr Soc, 57(7), 1175-1183.

Gorecki C., Nixon J., Madill A., Firth J. \& Brown J.M. (2012) What influences the impact of pressure ulcer on health-related quality of life? A qualitative patient-focused exploration of contributing factors. Journal of Tissue Viability, 21(1), 3-12.

He W., Liu P. \& Chen H.L., , (2012) The Braden Scale cannot be used alone for assessing pressure ulcer risk in surgical patients: a meta-analysis. Ostomy Wound Management, $58,34-40$.

Health Policy Brief (2015) Medicare's Hospital-Acquired Condition Reduction Program. The Centers for Medicare and Medicaid Services aims to decrease preventable conditions by reducing payments to the lowest-performing hospitals. Health Affairs August 6. Available at: http://healthaffairs.org/healthpolicybriefs/brief_pdfs/healthpolicybrief_142.pdf

Heinhold H., Westerfellhaus A. \& Kroger K. (2014) Prevalence of pressure ulcers in hospitalised patients in Germay - Trends from 2005 to 2011. EWMA Journal, 14(1), 9-14.

Horn S.D., Buerhaus P., Bergstrom N. \& Smout R.J. (2005) RN staffing time and outcomes of long-stay nursing home residents: pressure ulcers and other adverse outcomes are less likely as RNs spend more time on direct patient care. AJN The American Journal of Nursing, 105(11), 58-70.

HSCIC (2014) NHS Safety Thermometer Annual Publication - April 2012 to March 2014. Health and Social Care Information Centre.

Jones D. (2013) Pressure ulcer prevention in the community setting. Nursing Standard, 28(3), 47-55.

Kelechi T.J., Arndt J.V. \& Dove A. (2013) Review of pressure ulcer risk assessment scales. Journal of Wound Ostomy and Continence Nursing, 40(3), 232-236.

Lang T.A., Hodge M., Olson V., Romano P.S. \& Kravitz R.L. (2004) Nurse-patient ratios: a systematic review on the effects of nurse staffing on patient, nurse employee, and hospital outcomes. J Nurs Adm, 34(7-8), 326-337. 
Lyder C.H., Yun Wang N.D., Metersky M., Curry M., Kliman R., Verzier N.R. \& Hunt D.R. ( 2012) Hospital-acquired pressure ulcers: Results from the National Medicare Patient Safety Monitoring System Study. JAGS 60, 1603-1608.

Montalvo I. (2007) The National Database of Nursing Quality IndicatorsTM (NDNQI ${ }^{\circledR)}$. OJIN: The Online Journal of Issues in Nursing, 12(3).

Moore Z.E. \& Cowman S. (2015) Repositioning for treating pressure ulcers. status and date: New search for studies and content updated (no change to conclusions), published in, (1).

Mulligan S., Prentice J. \& Scott L. (2011) Prevalence Survey State-Wide Overview. WoundsWest Wound, Perth, WA.

Nguyen K.H., Chaboyer W. \& Whitty J.A. (2015) Pressure injury in Australian public hospitals: a cost-of-illness study. Australian Health Review.2 Epub ahead of print

NICE (2014) Pressure ulcers: prevention and management of pressure. NICE clinical guideline 179, Available at: http.guidance.nice.org.uk/cg179.

Niederhauser A., Lukas C.V., Parker V., Ayello E.A., Zulkowski K. \& Berlowitz D. (2012) Comprehensive programs for preventing pressure ulcers: a review of the literature. Advances in skin \& wound care, 25(4), 167-188.

NPUAP (2014) Pressure ulcer treatment recommendations.In: Prevention and treatment of pressure ulcers: clinical practice guideline. In Prevention of pressure ulcers. In: Prevention and treatment of pressure ulcers: clinical practice guideline (E.P.U.A.P. National Pressure Ulcer Advisory Panel, Pan Pacific Pressure Injury Alliance. ed.). pp. p. 42-78. National Pressure Ulcer Advisory Panel, Washington (DC).

Pinkney L., Nixon J., Wilson L., Coleman S., McGinnis E., Stubbs N., Dealey C., Nelson A., Patterson M. \& Keen J. (2014) Why do patients develop severe pressure ulcers? A retrospective case study. BMJ open, 4:e004303.

QIS NHS. (2009) Best Practice Statement: the Prevention and Treatment of Pressure Ulcers. Quality Improvement Scotland, National Health Service. Available at: http://www.healthcareimprovementscotland.org/

Rau, J., (2014) Medicare cuts payments to 721 hosptials with highest rates of infections, injuries. Kaiser Health News. Available at: http://khn.org/news/medicare-cutspayments-to-721-hospitals-with-highest-rates-of-infections-injuries/

Riordan J. \& Voegeli D. (2009) Prevention and treatment of pressure ulcers. British Journal of Nursing, 18(20), S20-S27.

Ritter, A., M. Livingston, J. Chalmers, L. Berends and P. Reuter (2016 early view) Comparative policy analysis for alcohol and drugs: current state of the field. International Journal of Drug Policy. doi:10.1016/i.drugpo.2016.02.004.Sen C.K., Gordillo G.M., Roy S., Kirsner R., Lambert L., Hunt T.K., Gottrup F., Gurtner G.C. \& Longaker M.T. (2009) Human Skin Wounds: A Major and Snowballing Threat to Public Health and the Economy. Wound repair and regeneration : official publication of the Wound Healing Society [and] the European Tissue Repair Society, 17(6), 763771.

Simonetti V., Comparcini D., Flacco M.E., Di Giovanni P. \& Cicolini G. (2015) Nursing students' knowledge and attitude on pressure ulcer prevention evidence-based guidelines: A multicenter cross-sectional study. Nurse education today, 35(4), 573579.

Soban L.M., Hempel S., Munjas B.A., Miles J. \& Rubenstein L.V. (2011) Preventing pressure ulcers in hospitals: A systematic review of nurse-focused quality improvement interventions. Joint Commission Journal on Quality and Patient Safety, $37(6), 245-245$. 
Spilsbury K., Nelson A., Cullum N., Iglesias C., Nixon J. \& Mason S. (2007) Pressure ulcers and their treatment and effects on quality of life: hospital inpatient perspectives. Journal of Advanced Nursing, 57(5), 494-504.

Still M.D., Cross L.C., Dunlap M., Rencher R., Larkins E.R., Carpenter D.L., Buchman T.G. \& Coopersmith C.M. (2013) The turn team: a novel strategy for reducing pressure ulcers in the surgical intensive care unit. Journal of the American College of Surgeons, 216(3), 373-379.

Stone P.W., Mooney-Kane C., Larson E.L., Horan T., Glance L.G., Zwanziger J. \& Dick A.W. (2007) Nurse working conditions and patient safety outcomes. Medical care, 45(6), 571-578.

Squires, M. A. E., Tourangeau, A. N. N. Spence Laschinger H. K. and Doran D. (2010). The link between leadership and safety outcomes in hospitals. Journal of Nursing Management 18(8): 914-925.

Sullivan N. \& Schoelles K.M. (2013) Preventing in-facility pressure ulcers as a patient safety strategy: a systematic review. Annals of Internal Medicine, 158(5_Part_2), 410-416.

Walt G., Shiffman J., Schneider H., Murray S.F., Brugha R. \& Gilson L. (2008) 'Doing' health policy analysis: methodological and conceptual reflections and challenges. Health Policy and Planning, 23, 308-317.

Weimer, D. L., \& Vining, A. (2015). Policy analysis: Concepts and practice. Routledge.

WOCN. (2010) Guidelines for Prevention and Management of Pressure Ulcers. Wound, Ostomy and Continence Nurses Society, NJ.

Zaratkiewicz S., Whitney J.D., Lowe J.R., Taylor S., O'Donnell F. \& Minton-Foltz P. (2010) Development and Implementation of a Hospital-Acquired Pressure Ulcer Incidence Tracking System and Algorithm. Journal for Healthcare Quality, 32(6), 44-51.

Zhu X.W., You L.M., Zheng J., Liu K., Fang J.B., Hou S.X., Lu M., L.A.1., Ma W.G. \& Wang H., (2012) Nurse staffing levels make a difference on patient outcomes: a multisite study in Chinese hospitals. Journal of Nursing Scholarship, 44(3), 266-273. 